\title{
Densidade Mineral Óssea de Mulheres na Pós-menopausa com e sem Antecedente de Histerectomia com Conservação Ovariana Bilateral
}

\author{
Bone Mineral Density in Postmenopausal Women with and without Previous \\ Hysterectomy with Bilateral Ovarian Conservation \\ João Daniel Hobeika, Aarão Mendes Pinto-Neto, Lúcia Helena Simões da Costa-Paiva \\ Adriana Orcesi Pedro, Edson Zangiacomi Martinez
}

\begin{abstract}
RESUMO
Objetivo: avaliar a densidade mineral óssea de mulheres na pós-menopausa com o antecedente cirúrgico de histerectomia com conservação ovariana bilateral, realizada no menacme, comparadas a um grupo de mulheres com menopausa natural, não-histerectomizadas.

Métodos: tratou-se de um estudo de corte transversal, com 30 mulheres histerectomizadas no menacme, avaliadas na pós-menopausa, comparadas a 102 mulheres menopausadas naturalmente, sendo realizado a densitometria óssea (Lunar DPX).

Resultados: as médias etárias, indice de massa corporal, cor da pele, tabagismo, escolaridade, menarca, paridade e antecedente de laqueadura tubária foram semelhantes nos grupos estudados. A comparação das médias da densidade mineral óssea e do T-score dos três locais do fêmur, utilizando o teste de Bonferroni, não apresentou diferenças estatisticamente significativas. As médias da densidade mineral óssea e do T-score da coluna lombar foram analisadas pelo teste t de Student e também não mostraram diferenças estatísticas.

Conclusão: estes resultados sugerem que a histerectomia com conservação ovariana bilateral, realizada em mulheres no menacme, não parece ocasionar redução adicional da massa óssea, quando avaliadas na pós-menopausa.
\end{abstract}

PALAVRAS-CHAVE: Densitometria óssea. Ovários. Osteoporose. Menopausa.

Introdução

A histerectomia é uma das cirurgias ginecológicas mais freqüentemente realizadas em mulheres adultas, nos países desenvolvidos. Estima-se que nos Estados Unidos, 20\% das mulheres de 40 anos estejam histerectomizadas, aumentando para $37 \%$ aos 65 anos $^{1}$. Entre 1988 e 1990, cerca de 1,7 milhões de mulheres foram

Trabalho realizado no Ambulatório de Menopausa do Departamento de Tocoginecologia da Faculdade de Ciências Médicas e Centro de Atenção Integral à Saúde da Mulher (CAISM) da Universidade Estadual de Campinas (UNICAMP) Correspondência:

Aarão Mendes Pinto-Neto

Assessoria Técnica e Científica do CAISM/UNICAMP

Rua Alexander Fleming, 101 - Cidade Universitária "Zeferino Vaz"

13.023-230 - Campinas - SP

Fone: (19) 788-9402

e-mail: jhobeika@cosmo.com.br

Este estudo recebeu apoio da Fundação Coordenação de Aperfeiçoamento de Pessoal de Nivel Superior (CAPES) histerectomizadas nos Estados Unidos, realizando-se ooforectomia bilateral no mesmo ato cirúrgico em $37 \%$ das mulheres com menos de 45 anos e em $68 \%$ quando maiores de 45 anos $^{2}$.

No Brasil, em 1996, foram realizadas 77.804 histerectomias financiadas pelo Sistema Único de Saúde (SUS), sendo a maioria por doenças benignas, principalmente a miomatose uterina $^{3}$. No município de Campinas a prevalência de mulheres histerectomizadas na faixa etária entre 45 e 60 anos é de $15,6 \%{ }^{4}$.

A função ovariana pós-histerectomia é um assunto muito controverso na literatura e na prática médica diária. Muitos pesquisadores descreveram modificações na função ovariana após a histerectomia, por alterações no aporte sangüíneo gonadal, podendo levar à menopausa mais precoce e conseqüentemente a uma perda mais acentuada da massa óssea ${ }^{5-9}$. Sendo a osteoporose e as fraturas as principais conseqüências tardias da falência ovariana, realizamos 
este estudo, que teve como objetivo principal avaliar as alterações na massa óssea em mulheres histerectomizadas menopausadas, quando comparadas às não-histerectomizadas.

\section{Pacientes e Métodos}

Foram avaliadas algumas características clinicas e a densitometria óssea (Lunar DPX) de 30 mulheres na pós-menopausa submetidas à histerectomia no menacme com conservação ovariana bilateral, (grupo "histerectomizadas"). Estas foram comparadas as 102 mulheres com menopausa natural (grupo "não-histerectomizadas"). As mulheres foram selecionadas no Ambulatório de Menopausa do Departamento de Tocoginecologia (DTG) do Centro de Atenção Integral à Saúde da Mulher (CAISM) da Universidade Estadual de Campinas (UNICAMP), no período entre setembro de 1997 e fevereiro de 1999.

No grupo "histerectomizadas" foram incluidas mulheres com o antecedente de histerectomia com conservação ovariana bilateral, idade entre 45 e 64 anos, cor branca ou parda e com dosagem de FSH plasmático $>30 \mathrm{UI} / \mathrm{mL}$, confirmando a menopausa. No grupo "não-histerectomizadas" incluímos mulheres com útero e ovários preservados, com a mesma faixa etária e cor da pele e que apresentassem amenorréia há pelo menos 12 meses.

Excluímos as mulheres que apresentassem doenças, tais como o diabetes mellitus e as alterações tiroideanas, e também as usuárias de medicamentos que sabidamente alteram o metabolismo ósseo. Também excluímos aquelas com antecedente de radioterapia ou quimioterapia prévia, as tabagistas de mais de 20 cigarros/dia, as etilistas com ingestão de mais de dois copos de bebida alcóolica/dia e, finalmente, as mulheres que apresentassem um indice de massa corporal (IMC) $<20 \mathrm{~kg} / \mathrm{m}^{2}$ ou $>30 \mathrm{~kg} / \mathrm{m}^{2}{ }^{10}$.

A variável independente estudada foi a histerectomia com conservação ovariana bilateral, ao passo que a variável dependente foi a densidade mineral óssea (DMO), sendo expressa em valores absolutos em $\mathrm{g} / \mathrm{cm}^{2}$ e valores relativos em $T$-score (adulto jovem). Segundo os critérios da Organização Mundial da Saúde de $1994^{11}$, os resultados da densitometria óssea são classificados em normal, osteopenia e osteoporose. Neste estudo, esta variável foi categorizada em normal (valores de T-score maiores ou iguais a -1 DP) ou alterada (valores de T-score inferiores a de -1 DP).

Como variáveis de controle foram analisa- das: idade, IMC, cor da pele, escolaridade, tabagismo, idade à menarca, paridade, antecedente de laqueadura tubária, tempo de amenorréia, tempo de histerectomia e o tempo de sintomas climatéricos.

Inicialmente, foram elaboradas tabelas descritivas, utilizando-se freqüência, média e desvio-padrão, sendo a comparação da médias realizada por meio do teste $t$ de Student. Em situações em que os valores tivessem uma distribuição não-normal, não permitindo comparar médias, utilizou-se o teste não-paramétrico de Mann-Whitney. Para a comparação das freqüências foram utilizados os testes exato de Fisher ou $\chi^{2}$ de Pearson ${ }^{12}$.

Para a comparação das médias da DMO em $\mathrm{g} / \mathrm{cm}^{2}$ e dos valores relativos em $T$-score no fêmur, foi utilizado o teste de Bonferroni, que faz uma comparação múltipla dos três locais analisados ${ }^{13}$. Para a coluna lombar utilizou-se o teste $t$ de Student para amostras independentes. A análise da distribuição segundo categorias de T-score normal ou alterada, de acordo com os locais analisados, foi realizada utilizando-se o teste $\chi^{2}$ de Pearson ${ }^{14}$. Esta pesquisa foi aprovada pela Comissão de Pesquisa do CAISM/Unicamp e Comitê de Ética da FCM/Unicamp.

\section{Resultados}

A Tabela 1 apresenta algumas características clínicas das mulheres dos dois grupos, mostrando que não houve diferenças estatisticamente significativas em relação a estas variáveis.

A análise estatística entre o tempo médio de amenorréia das mulheres não-histerectomizadas (3,9 anos) e o tempo médio dos sintomas climatéricos nas mulheres histerectomizadas $(4,2$ anos) não mostrou diferença significativa. As mulheres do grupo "histerectomizadas" haviam sido submetidas a esta cirurgia há 12 anos em média, com variação entre 1 e 25 anos. A média etária destas mulheres, na época da histerectomia, foi de 41 anos (Tabela 1).

A comparação das médias das DMO em g/ $\mathrm{cm}^{2}$, avaliadas no nível do colo do fêmur, triângulo de Wards, trocânter e coluna lombar $\left(\mathrm{L}_{2}-\mathrm{L}_{4}\right)$, não mostrou diferenças significativas entre os dois grupos (Tabela 2).

Em relação aos valores médios do $T$-score, não houve diferenças estatísticas, tanto no fêmur como na coluna lombar (Tabela 3).

$\mathrm{Na}$ Tabela 4, os valores de T-score foram agrupados em normal, quando apresentavam valores superiores a $-1 \mathrm{DP}$, e alterado, quando 
inferiores a -1 DP. Não houve diferenças significativas na porcentagem de densitometrias nor- mais e alteradas nos dois grupos, tanto nos três locais do fêmur, como na coluna lombar.

Tabela 1 - Características clínicas e gineco-obstétricas das mulheres histerectomizadas e não-histerectomizadas.

\begin{tabular}{|c|c|c|c|}
\hline Variáveis & $\begin{array}{l}\text { Histerectomizadas } \\
\qquad n=30\end{array}$ & $\begin{array}{l}\text { Não-histerectomizadas } \\
\qquad n=102\end{array}$ & $\mathrm{p}$ \\
\hline Idade $\left(\right.$ anos) ${ }^{*}$ & $53,1 \pm 5,1$ & $52,6 \pm 4,0$ & $0,61^{(1)}$ \\
\hline $\operatorname{IMC}\left(\mathrm{kg} / \mathrm{m}^{2}\right)^{*}$ & $25,8 \pm 3,2$ & $26,4 \pm 3,6$ & $0,41^{(1)}$ \\
\hline Escolaridade (anos) ${ }^{*}$ & $4,6 \pm 4,3$ & $4,3 \pm 3,5$ & $0,97^{(2)}$ \\
\hline Brancas (\%) & 83,3 & 93,1 & $0,14^{(3)}$ \\
\hline Tabagistas (\%) & 23,3 & 13,7 & $0,26^{(3)}$ \\
\hline Menarca (anos) ${ }^{*}$ & $12,9 \pm 2,2$ & $13,3 \pm 1,6$ & $0,24^{(2)}$ \\
\hline Paridade* & $3,8 \pm 2,6$ & $3,9 \pm 2,5$ & $0,96^{(2)}$ \\
\hline Laqueadura tubária \% & 23,3 & 38,2 & $0,13^{(4)}$ \\
\hline Tempo de amenorréia $(\operatorname{anos})^{*}$ & & $3,9 \pm 3,2$ & $* *(2)$ \\
\hline Tempo de sintomas (anos) $)^{*}$ & $4,2 \pm 4,5$ & & $* *(2)$ \\
\hline Tempo de histerectomia (anos) $)^{*}$ & $12,1 \pm 7,7$ & & \\
\hline Idade à histerectomia (anos) ${ }^{*}$ & $41,0 \pm 7,3$ & & \\
\hline
\end{tabular}

* Média \pm DP

** Comparação entre o tempo de amenorréia do grupo não-histerectomizadas e o tempo de sintomas climatéricos do grupo histerectomizadas, $p=0,32$.

(1) Teste $t$ de Student para comparação de médias

(2) Teste não-paramétrico de Mann-Whitney

(3) Teste exato de Fisher

(4) Teste $\chi^{2}$ de Pearson

Tabela 2 - Médias da DMO $\left(\mathrm{g} / \mathrm{cm}^{2}\right)$ do fêmur e coluna lombar, nos grupos de mulheres histerectomizadas e não-histerectomizadas.

\begin{tabular}{lccccc}
\hline Local & \multicolumn{2}{c}{ Histerectomizadas } & \multicolumn{2}{c}{ Não-histerectomizadas } & $\mathbf{p}$ \\
& \multicolumn{2}{c}{$\mathbf{n = 3 0}$} & \multicolumn{2}{c}{$\mathbf{n = 1 0 2}$} & \\
& Média & DP & Média & DP & \\
\hline Colo & 0,906 & 0,139 & 0,922 & 0,130 & $*(1)$ \\
Wards & 0,772 & 0,171 & 0,785 & 0,142 & $*(1)$ \\
Trocânter & 0,733 & 0,116 & 0,764 & 0,125 & $*(1)$ \\
$\mathrm{L}_{2}$-L 4 & 1,098 & 0,144 & 1,064 & 0,149 & $0,28^{(2)}$ \\
\hline
\end{tabular}

* Comparação múltipla das médias da DMO avaliadas nos três locais do fêmur, $p=0,46$

(1) Teste de Bonferroni

(2) Teste $t$ de Student para amostra independentes
Tabela 3 - Valores médios de T-score no fêmur e coluna lombar, nos grupos de mulheres histerectomizadas e não-histerectomizadas.

\begin{tabular}{|c|c|c|c|c|c|}
\hline \multirow[t]{2}{*}{ Local } & \multicolumn{2}{|c|}{$\begin{array}{l}\text { Histerectomizadas } \\
\qquad n=30\end{array}$} & \multicolumn{2}{|c|}{$\begin{array}{c}\text { Não-histerectomizadas } \\
n=102\end{array}$} & \multirow[t]{2}{*}{$p$} \\
\hline & Média & DP & Média & DP & \\
\hline Colo & $-0,615$ & 1,167 & $-0,472$ & 1,093 & $*(1)$ \\
\hline Wards & $-1,060$ & 1,317 & $-0,955$ & 1,097 & $*(1)$ \\
\hline Trocânter & $-0,512$ & 1,055 & $-0,236$ & 1,139 & * (1) \\
\hline $\mathrm{L}_{2}-\mathrm{L}_{4}$ & $-0,864$ & 1,232 & $-1,119$ & 1,250 & $0,33^{(2)}$ \\
\hline
\end{tabular}

* Comparação múltipla das médias do T-score avaliadas nos três locais do fêmur, $p=0,47$

(1) Teste de Bonferroni

(2) Teste $t$ de Student para amotras independentes
Tabela 4-Distribuição percentual das mulheres histerectomizadas enão-histerectomizadas, segundo categorias de $T$-score do fêmur e coluna lombar.

\begin{tabular}{lccccc}
\hline Local & \multicolumn{2}{c}{$\begin{array}{c}\text { Histerectomizadas } \\
\mathbf{n = 3 0}\end{array}$} & \multicolumn{2}{c}{$\begin{array}{c}\text { Não-histerectomizadas } \\
\mathbf{n = 1 0 2}\end{array}$} & $\mathbf{p}$ \\
& Normal & Alterado & Normal & Alterado & \\
\hline Colo & 66,7 & 33,3 & 60,8 & 39,2 & 0,63 \\
Wards & 56,7 & 43,3 & 47,0 & 53,0 & 0,36 \\
Trocânter & 66,7 & 13,3 & 72,5 & 27,5 & 0,53 \\
$\mathrm{~L}_{2}-\mathrm{L}_{4}$ & 53,3 & 46,7 & 41,2 & 58,8 & 0,24 \\
\hline
\end{tabular}

Teste $\chi^{2}$ de Pearson

\section{Discussão}

Os resultados deste estudo mostraram não haver alteração na massa óssea de mulheres histerectomizadas no menacme com conservação ovariana bilateral, quando avaliadas na pós-menopausa, comparadas a um grupo de mulheres também na pós-menopausa, sem o antecedente de histerectomia. Este resultado foi semelhante ao relatado por Modesto Filho et al. ${ }^{15}$, que ob- 
servaram que a histerectomia com conservação ovariana uni ou bilateral não acarretou aumento da perda óssea, além do normalmente esperado.

Por outro lado, os resultados deste estudo foram contraditórios em relação aos reportados por Hreshchyshyn et al. ${ }^{5}$ nos Estados Unidos, e por Watson et al. ${ }^{7}$ na Inglaterra. Estes dois estudos descreveram diminuição da massa óssea em mulheres após a histerectomia com conservação ovariana. Menon et al. ${ }^{16}$, também na Inglaterra, relatam que mulheres histerectomizadas com conservação ovariana apresentam quadro de hipoestrogenismo suficiente para causar ondas de calor, perda óssea e elevação das concentrações sangüineas de ácido úrico.

O presente estudo difere dos citados na literatura revisada, pelo fato de os grupos terem sido controlados por várias características que sabidamente poderiam influenciar o resultado final. Segundo Dean ${ }^{17}$, as pesquisas publicadas, apesar de controlarem as mulheres por idade, duração dos sintomas climatéricos e indice de massa corporal, falharam por não considerarem os hábitos e principalmente o passado reprodutivo das pacientes.

Em relação aos fatores reprodutivos, não observamos diferenças nas mulheres histerectomizadas, em relação ao grupo de referência. Apesar da influência de muitas destas variáveis sobre a massa óssea não estar claramente estabelecida, algumas adquirem especial importância pela sua alta prevalência. A laqueadura tubária, por exemplo, constitui um problema de saúde pública ${ }^{18} \mathrm{e}$, mais ainda, pode alterar o aporte sangüíneo ovariano, influenciando a massa óssea ${ }^{19}$. Neste estudo, o percentual de mulheres não-histerectomizadas e com o antecedente de laqueadura tubária foi maior em relação às mulheres histerectomizadas, porém sem diferença estatisticamente significativa. Este resultado foi esperado, pois as mulheres que foram submetidas à histerectomia não necessitam de laqueadura tubária futura.

Cabe citar também que a média etária das mulheres avaliadas foi de aproximadamente 53 anos, sendo semelhante entre os grupos. A média etária à histerectomia foi de 41 anos, mas os sintomas climatéricos somente apareceram por volta dos 49 anos, idade esta compativel com a maioria dos estudos que avaliaram a idade de ocorrência de menopausa natural em nosso meio $^{20-22}$. No município de Campinas, um estudo recente de base populacional mostrou que aos 53 anos, aproximadamente $80 \%$ das mulheres já estão na pós-menopausa, e que a média etária de ocorrência da menopausa é de 47,5 anos ${ }^{4}$.
Isto pode ser considerado indicativo de que a histerectomia, além de não ter alterado a massa óssea, não causou falência ovariana prematura, em concordância com os resultados de Lima et $a .^{23}$, que avaliaram a função ovariana de 20 mulheres histerectomizadas no menacme e não observaram alterações endócrinas ou ultrasonográficas após a cirurgia.

Nas mulheres histerectomizadas, a confirmação da conservação ovariana bilateral baseou-se na informação da mulher ou na presença de laudo anatomopatológico referindo somente a retirada do útero. A ultra-sonografia não foi utilizada para a confirmação da presença dos ovários, pois nas mulheres na pós-menopausa ocorre uma esperada atrofia ovariana, dificultando em muito a detecção ecográfica. Se tivéssemos incluído neste estudo somente mulheres com ovários visiveis à ultra-sonografia, estaríamos cometendo um viés de seleção, pois estaríamos excluindo aquelas que apresentassem ovários atróficos ${ }^{24}$.

Reconhecemos como a principal limitação na interpretação dos resultados deste estudo o fato de não se tratar de uma pesquisa prospectiva, com medidas da densidade mineral óssea antes e depois da histerectomia. Também pode ser que tenhamos avaliado o efeito final da histerectomia com conservação ovariana bilateral, pois, segundo Kritz-Silverstein e BarrettConnor $^{25}$, apesar da perda óssea ser mais rápida após a ooforectomia bilateral, a massa óssea final é igual em mulheres ooforectomizadas ou não. Estes autores também verificaram que mulheres histerectomizadas que utilizaram terapia de reposição hormonal possuem maior massa óssea, independentemente da ooforectomia.

\section{SUMMARY}

Purpose: to evaluate the bone mineral density of postmenopausal women with previous hysterectomy and with bilateral ovarian conservation compared to a group of nonhysterectomized naturally menopausal women.

Methods: this is a cross-sectional study of bone densitometry (Lunar DPX) in 30 menopausal women hysterectomized when in the premenopause compared with 102 naturally postmenopausal women.

Results: the mean age, body mass index, color of the skin, smoking habits, educational level, menarche, parity and previous tubal ligation were similar in the studied groups. Bone mineral density average and the T-score of the three femural sites analyzed by the Bonferroni test did not show significant differences. The bone mineral density average and the T-score of the lumbar spine were analyzed by the Student $\mathrm{t}$ test and did not show statistical differences. 
Conclusion: these findings suggest that premenopausal hysterectomy with bilateral ovarian conservation does not cause an additional reduction in bone mineral content when evaluated in the postmenopause.

KEY WORDS: Bone densitometry. Ovaries. Osteoporosis. Menopause.

\section{Referências}

1. Pokras R. Hysterectomy: past, present, and future. Stat Bull Metrop Insur Co 1989; 70:12-21.

2. Wilcox LS, Koonin LM, Pokras R, Strauss LT, Xia Z, Peterson HB. Hysterectomy in the United States, 1988-1990. Obstet Gynecol 1994; 83:549-55.

3. O’ Dwyer G. Avaliação da qualidade da assistência prestada a mulheres submetidas a histerectomia por leiomioma [dissertação]. Rio de Janeiro: Fundação Oswaldo Cruz; 1998.

4. Pedro AO, Pinto-Neto AM, Hardy EE, Costa-Paiva LHS, Osis MJC, Lane E. Age at menopause and perception of climacterics symptoms among Brazilian women living in Campinas, São Paulo State, Brazil. Menopause 1998; 5:269-71.

5. Hreshchyshyn MM, Hopkins A, Zylstra S, Anbar M. Effects of natural menopause, hysterectomy and oophorectomy on lumbar spine and femural neck bone densities. Obstet Gynecol 1988; 72:631-8.

6. Souza AZ, Fonseca AM, Izzo VM, Clauze RM, Salvatore CA. Ovarian histology and function after total abdominal hysterectomy. Obstet Gynecol 1986; 68:847-9.

7. Watson NR, Studd JW, Garnett T, Savvas M, Milligan P. Bone loss after hysterectomy with ovarian conservation. Obstet Gynecol 1995; 86:72-7.

8. Oldenhave A, Jaszmann LJ, Everaerd WT, Haspels AA. Hysterectomized women with ovarian conservation report more severe climacteric complaints than do normal climacteric women of similar age. Am J Obstet Gynecol 1993; 168:765-71.

9. Siddle N, Sarrel P, Whitehead M. The effect of hysterectomy on the age at ovarian failure: identification of a subgroup of woman with premature loss of ovarian function and literature review. Fertil Steril 1987; 47:94-100.

10.Peris P, Guañabens N, Parés A, et al. Vertebral fractures and osteopenia in chronic alcoholic patients. Calcif Tissue Int 1995; 57:111-4.

11.WHO. World Health Organization. Assessment of fracture risk and its application to screening for postmenopausal osteoporosis. Geneva: WHO; 1994. 130p. (Technical report series, 843).
12.Beiguelman B. Curso prático de bioestatística. $2^{a}$ ed. Ribeirão Preto: Sociedade Brasileira de Genética; 1991. 231p.

13.Johnson RA, Wichern DW. Applied multivariate statistical analysis. $3^{\text {rd }}$ ed. Englewood Cliffs: Prentice-Hall; 1992. p.219-84.

14.Agresti A. Categorical data analysis. New York: John Wiley \& Sons; 1990. 558p.

15. Modesto Filho J, Londres SJB, Ferreira-Filho JA, Vasconcelos THC, Azevedo LCP, Azevedo LAP. Efeito da ooforectomia e histerectomia sobre a densidade mineral óssea. Rev Bras Ginecol Obstet 1996; 18:123-8.

16. Menon RK, Okonofua FE, Agnew JE, et al. Endocrine and metabolic effects of simple hysterectomy. Int J Gynaecol Obstet 1987; 25:459-63.

17. Dean S. Hysterectomy and bone mineral density. Br J Hosp Med 1997; 57:207-9.

18.Carranza-Lira S, Murillo-Uribe A, Martínez-Trejo N, Santos-González J. Influence of previous bilateral partial tubal resection on symptoms, hormones, lipids, and bone density in postmenopausal women. Contraception 1997; 56:5-7.

19.Fox KM, Cummings SR. Is tubal ligation a risk factor for low bone density and increased risk of fracture? Am J Obstet Gynecol 1995; 172:1015 .

20.Fonseca AM, Hegg R, Guarnieri-Neto C, Melo NR, Filassi JR, Salvatore CA. Climatério: aspectos epidemiológicos e clínicos. Rev Bras Clin Ter 1985; 14:389-91.

21. Halbe HW, Fonseca AM, Assis JS, et al. Aspectos epidemiológicos e clínicos em 1319 pacientes climatéricas. Rev Ginecol Obstet 1990; 1:18294.

22.Wehba S, Fernandes CE, Melo NR, Ferreira JAS, Faria-Júnior D, Roucourt S. Aspectos epidemiológicos, clínicos e de diagnósticos do climatério. In: Fernandes CE, Melo NR, Soares CN, Wehba S. Hormonioterapia e Psicofarmacologia na Saúde da Mulher. São Paulo: SOBRAC; 1998. p.15-22.

23.Lima MFP, Reis RM, Bonduki CE, Zamoner MRF, Silva CS, Salum R. Análise da função ovariana em mulheres histerectomizadas. Rev Bras Med Ginecol Obstet 1996; 7:203-8.

24.Pinto-Neto AM, Pedro AO, Costa-Paiva LHS, et al. O papel do exame ecográfico na assistência à mulher na pós-menopausa, com ênfase no diagnóstico de tumor anexial. J Bras Ginecol 1997; 107:63-7.

25.Kritz-Silverstein D, Barrett-Connor E. Oophorectomy status and bone density in older, hysterectomized women. Am J Prev Med 1996; 12:424-9. 